
\title{
25 Research Suare \\ Debridement, Antibiotics and Implant Retention (DAIR) Has High Success Rate for Acute Periprosthetic Joint Infection
}

\section{Chaofan Zhang}

First Affiliated Hospital of Fujian Medical University https://orcid.org/0000-0001-7397-2298

\section{Long He}

First Affiliated Hospital of Fujian Medical University

\section{Xinyu Fang}

First Affiliated Hospital of Fujian Medical University

\section{Zida Huang}

First Affiliated Hospital of Fujian Medical University

\section{Guochang Bai}

First Affiliated Hospital of Fujian Medical University

\section{Wenbo Li}

First Affiliated Hospital of Fujian Medical University

Wenming Zhang ( $\boldsymbol{Z}$ zhangwm0591@fjmu.edu.cn )

https://orcid.org/0000-0003-1567-7279

\section{Research article}

Keywords: Debridement, antibiotics and implant retention (DAIR); acute periprosthetic joint infection, debridement, total knee replacement, total hip replacement

Posted Date: September 10th, 2019

DOI: https://doi.org/10.21203/rs.2.14208/v1

License: (a) (1) This work is licensed under a Creative Commons Attribution 4.0 International License. Read Full License 


\section{Abstract}

Background: The efficacy of debridement, antibiotics and implant retention (DAIR) for acute periprosthetic joint infection (PJI) has been debated. The purpose of this study was to report our clinical outcome and experience of managing acute PJI with DAIR.

Methods: A retrospective review of all patients diagnosed with acute PJI after hip or knee replacement surgery and managed with DAIR in our prospective joint replacement register from 2008 to 2019 was performed. Patients' symptom onset duration, inflammatory marker levels, bacteriology, and surgical outcome were tracked and recorded.

Results: A total of 24 patients with 7 after hip replacement and 17 after knee replacement were included. Twenty-one were early post-operative infection and 3 were late acute haematogenous infection. During a mean follow-up time of $29.2 \pm 15.1$ months, twenty-two were successfully treated while 2 failed who required repeated DAIR. The overall success rate of DAIR was $91.7 \%$. For staphylococcal infection, DAIR has $100 \%$ success rate. Five patients presented with symptoms between 4 to 8 weeks also achieved $100 \%$ success rate.

Conclusions: DAIR has a high success rate for acute PJI, and can be performed in selected patients whose symptoms have sustained for over 4 weeks. DAIR has high rate of success for staphylococcal infection. Metagenomic next-generation sequencing ( $m$ NGS) test can be used as a potential tool to identify pathogens in acute PJI. Key Words: Debridement, antibiotics and implant retention (DAIR); acute periprosthetic joint infection, debridement, total knee replacement, total hip replacement

\section{Background}

Periprosthetic joint infection (PJI) after total hip and total knee arthroplasty is a rare but catastrophic complication that can cause significant morbidity to the patient and cost to the health care system [1]. It was found from the Medicare inpatient data set that the 1-year and 5-year risk of PJI was $0.69 \%$ and $1.09 \%$ for THA and $0.74 \%$ and $1.38 \%$ for TKA, respectively [2]. Though not commonly seen, treating PJI has already been estimated to cost the USA $\$ 566$ million in 2009 , and the number is projected to reach $\$ 1.62$ billion in 2020 [1].

Acute PJI, as proposed by Tsukayama, is defined as infections happening within 4 weeks from the primary arthroplasty, or acute onset of symptoms within 4 weeks in a previously well-functioning joint (hematogenous infection) [3]. In the past few decades, debridement, antibiotics and implant retention (DAIR), which primarily included a thorough debridement and exchange of the polyethylene inserts or femoral head, has been widely accepted as the first-line treatment for acute PJI. However, literature showed various success rate ranging from $26 \%$ to $92 \%$ [3-17].

There have been several risk factors proposed to affect the outcome of DAIR, including age, American Society of Anesthesiologists (ASA) score, diabetes mellitus (DM), rheumatoid arthritis, and steroid 
therapy [18]. On the other hand, the role of optimal timing of DAIR, antibiotic regime, the involved microorganism, and whether or not to exchange the polyliner are still unclear. Therefore the purpose of this study is to report our outcome and experience of managing acute PJI with DAIR.

\section{Methods}

A retrospective review of our prospective joint replacement registry from 2008 to 2019 was carried out. Patients who were diagnosed with acute PJI after hip or knee replacement surgery and managed with DAIR were included. The diagnosis of PJI was based on the 2011 Musculoskeletal Infection Society (MSIS) PJI diagnostic criteria [19]. Both the early post-operative infections (Tsukayama type 2) and the late haematogenous infections (Tsukayama type 3) were included [3]. 'Acute' was defined as infections happening within 3 months after the primary surgery, or symptom onset within 3 months in a late wellfunctioning joint, as was proposed by Zimmerli [20].

Our center was a tertiary referral center in Fujian province, which has a population of 38.6 million. Most patients received joint replacement surgery in other hospitals but were transferred to our center for further management. Once infection was suspected, blood tests including C-Reactive Protein (CRP), Erythrocyte Sedimentation Rate (ESR) and White Blood Cell (WBC) were ordered. Joint aspiration was performed to collect synovial fluid and sent for WBC and differential count, as well as microbiological culture. For hip infections, aspiration was carried out under ultrasound guidance.

Once patient was diagnosed with acute PJI, empirical antibiotics were used and DAIR was performed as soon as possible, regardless of the microorganism culture results. Surgery was performed by the single senior surgeon (W Zhang) using a standard operating setup. Generally spinal anesthesia was performed. Surgical site was disinfected 3 times with tincture of iodine followed by $70 \%$ alcohol. Previous incision was used and the superficial wound was debrided. Before opening up the synovial cavity, aspiration was performed again to collect synovial fluid and sent for culture or metagenomic next-generation sequencing (mNGS) test. Once the cavity was cut open, at least five separated periprosthetic tissue samples were collected with surgical knife cut and sent for microbiological culture and mNGS. The polyethylene insert or femoral head was then taken out and systematic debridement was performed to remove all the infected or necrotic tissues. Would was soaked in iodine solution for $30 \mathrm{mins}$ and irrigated with 3 liters of saline using a pulsed lavage gun. Would was closed temporally and re-draped, and a new set of surgical tools was used. After re-disinfecting the surgical site, the new insert/femoral head was placed in and would was closed with 3-layer closure. Drain was usually kept for 2 days. Tourniquet was used if too much bleeding was encountered during knee debridement.

Culture has also been a standardized procedure in our center. Joint fluid, once collected, was injected into aerobic, anaerobic bottles, and inoculated to fungal plate. A small amount of fluid was also injected into pediatric blood culture bottle. For tissues, they were collected by blade cut instead of electrode, and further cut into pieces, stored into sterile container and sent for culture immediately from operation theater to the department of microbiology by designated personnel. Culture was routinely performed for 7 
days, but in case of negative results or suspected low-virulence pathogen, culture was prolonged to 14 days. A paired sample of joint fluid and tissues were also sent for mNGS exam, which was carried out by the BGI group company (Shenzhen, China). The results were normally available in 48 hours.

An empirical antibiotics regime of vancomycin combined with ceftazidime were prescribed until culture/mNGS results were available. It was then changed to pathogen-specific antibiotics according to the drug susceptibility results. In general circumstances, intravenous (IV) antibiotics were used for 2 weeks followed by oral antibiotics for additional 4 weeks.

Surgical outcomes were defined as successful if patients' clinical symptoms had been relieved, and inflammatory marker levels including CRP, ESR and WBC count had returned to normal, X-rays showed no prosthetic loosening, and no lifelong antibiotics suppression was required, at a minimum of one-year follow-up. Outcomes were defined as failed if patients required any further surgeries (e.g., additional DAIR or any forms of debridement, or one or two-stage revision), or needed lifelong antibiotics suppression.

All the patients' baseline characteristics were documented, including age, primary diagnosis, time interval of primary TKA to symptoms, time lag between symptoms onset and debridement, pre-operative CRP, ESR, WBC, lymphocyte, bacteriology, and synovial total cell count. The blood samples for inflammatory markers including CRP, ESR, and WBC were specified as being taken within 24 hours before debridement.

Paired-samples t-test (for parametric data) was used to compare the differences of inflammatory markers before and after surgery using the SPSS software (v22.0, IBM, USA). Statistical significance was assumed if the $p$ value was less than 0.05 .

\section{Results}

A total of 27 patients were tracked from our registry. One patient died from gastric cancer at 18 months after surgery, and 2 patients lost to follow-up. These patients were excluded from the study leaving 24 included in the final analysis. 13 patients were male and 11 were female, with a mean age of $63.8 \pm 13.3$ years and BMI of 25.6 \pm 3.5 . Seven were infections after hip replacement and 17 were after knee replacement. Hip replacements, all THA, were performed for various reasons including 2 femoral neck fracture, three osteonecrosis of femoral head (ONFH), and one ankylosing spondylitis (AS). For knee replacement surgery, 16 were TKA and 1 was unicondylar knee replacement (UKA). All the cases were performed for knee OA. 21 were early post-operative infections and 3 were hematogenous infections. 18 patients had comorbidity of hypertension, 7 with DM, 2 with AS, 2 with gout, 1 with hepatic cirrhosis, and 1 with varicose veins.

For presentation, two patients had sinus tract, and other patients complained mostly of pain, swelling or fever. The mean symptom onset time was 21.5 days. Five patients presented with symptoms for over 4 weeks but were still managed with DAIR. The mean synovial WBC was $20,107.5^{\star} 10^{\wedge} 6 / \mathrm{L}$ and percentage of polymorphonuclear neutrophils (PMN) was $85.3 \pm 7.2 \%$. For all DAIR, polyethylene insert/femoral head was exchanged during surgery. 
The mean follow-up time was $29.2 \pm 15.1$ months. Twenty-two cases were successful and 2 cases were failed. The overall success rate was $91.7 \%$. At the last follow-up, the mean CRP level decreased from $52.6 \pm 34.0$ to $5.4 \pm 3.5(p<0.001)$ and ESR from $72.3 \pm 34.3$ to $20.2 \pm 12.1(p<0.001)$ respectively. For knee infections, the KSS improved from $44.83 \pm 12.19$ to $81.42 \pm 9.18(p<0.001)$, and functional score from $38.09 \pm 3.47$ to $73.86 \pm 22.99(p<0.001)$. For hip infections, the HHS score improved from $34.41 \pm 6.85$ to $84.12 \pm 15.14(p<0.001)$. For the failed two cases, both patients received repeated DAIR, and no recurrent infections were seen.

It was noted that in the 19 cases in which DAIR was performed within 4 weeks, 17 were successful with success rate standing at $89.4 \%(17 / 19)$. In the other 5 cases, patients had sustained symptoms between 4 weeks to 8 weeks, with an average of 43.4 days, were still performed with DAIR and achieved a $100 \%$ success rate.

For bacteriology, culture was positive in 21 cases (87.5\%). There were 9 Methicillin-resistant Staphylococcus epidermidis (MRSE), 3 Staphylococcus aureus, 3 Streptococcus agalactiae, 1 Staphylococcus haemolyticus, 1 Streptococcus agalactiae subsp. equisimilis, 1 Streptococcus gordonii, 1 Bacteroides, 1 Enterococcus faecalis, and 1 Klebsiella pneumoniae. mNGS were performed in 10 cases with all positive results. Six yielded exactly the same pathogen as culture, but 2 were totally different. In one case, culture yields Klebsiella pneumoniae infection but mNGS also identified Bacteroides and Pseudomonas aeruginosa. In another case, culture was negative but mNGS identified Streptococcus agalactiae infection (Table 1).

The combined bacteriology results were described in Figure 1, with an overall pathogen identification rate of $91.7 \%(22 / 24)$. The success rate for Staphylococcus, Streptococcus, and other pathogen was $100 \%$ (9/9), 71.4\% (5/7), and 100\% (6/6) respectively. Both the 2 failed cases were infected with Streptococcus agalactiae.

\section{Discussion}

The management of acute PJI presents great challenge to orthopaedic surgeons. To extract the implant and perform a one-stage or two-stage revision may be a hard choice for both the patients and surgeons. Thus DAIR, which retains the protheses, remains the mainstay treatment for acute PJI. However, its efficacy has been debated during the past few decades. Several studies have reported an overall success rate of lower than $50 \%[11,12,14-17,21-23]$. (Table 2 )

A systematic review by Silva, which included 30 articles with 530 DAIR for PJI following TKA, revealed the success rate of only $32.6 \%$ after a mean 4 -year follow-up [30]. A similar research by Romano at 9 years later including 14 article and 710 acute PJI cases found the success rate was still not high, standing at $46 \%$ [31]. DAIR was therefore concerned because a failed DAIR would increase the likelihood of failure of the subsequent two-stage revision [32, 33]. From the recent 2018 international concensus meeting (ICM), $96 \%$ of delegates agree that the effectiveness of DAIR is still unclear due to lack of comparative data among the treatment options, and limited evidence to suggest superiority of any one treatment. The 
treatment decision must be made on a case-by-case basis and account for underlying medical conditions, infection history, organism characteristics, and surgical history. DAIR is most appropriate for acute PJI without complicating factors, such as extensive and pervasive infection by a high virulence or resistant organism [34].

This is a single-surgeon series reporting the outcome of standardized DAIR procedure for acute PJI. The main finding is DAIR has an overall high success rate for acute PJI. Despite the general not high success rate, however, after a deep review of the literature we found great heterogeneity of the included population. In some reports, the polyethylene inserts/femoral heads were not exchanged during DAIR. This may lead to incomplete debridement as the surgeon would have no access to the posterior joint capsule and bilateral gutters. Along with the biofilm adhered to the insert/femoral head, keeping it would definitely increase the bioburden of infection. Also, surgeons would have the chance to clean the tibial/femoral component once the insert or head is removed. Second, in some other reports, surgeries were performed by different surgeons with various experience and surgical skills. DAIR is an urgent surgery which requires a radical debridement of all the infected tissues and a standardized operating procedure should be set up. Finally, for most research reported, the culture positive rate was not high. This may increase the likelihood of failure because no pathogen-specific antibiotics could be used. In our case series, all the DAIR procedures were performed by the single experienced senior surgeon using standardized surgical procedure. The culture positive rate was as high as $90 \%$ following a strictly standardized culture protocol, and with the help of mNGS technique, we were able to identify pathogens in over $90 \%$ of patients and chose pathogen-specific antibiotics according to the drug-resistant results.

NGS, especially mNGS, is an evolving technology widely applied in clinical diagnosis [35]. mNGS sequences all nucleic acid fragments in a clinical sample, enabling the use of bioinformatics method to obtain microbial sequences and species information and therefore identifies the pathogen. Recently, owing to the substantial cost reduction, mNGS has also been increasingly applied to the diagnosis of bone and joint infection [36]. Our previous reports have shown that mNGS has potential in identifying pathogens even in cases with antibiotic treatment and in cases with rarely-seen pathogens [37, 38]. In this limited cohort of patients, mNGS also identified additional microorganisms in 2 cases, which added another evidence that mNGS may serve as a potential fast tool to diagnose PJI. However, more studies are warranted to prove its true efficacy. In our current practice, mNGS has been used as a routine diagnostic tool for suspected bone and joint infections.

Our second finding was DAIR could be considered even in patients whose symptoms were sustained for over 1 month.In our series, five cases were presented with symptom onset between 4 weeks to 8 weeks. These patients were all managed with DAIR and we saw no infection relapse during a minimum one-year follow up. A similar study was reported by Ottesen et al, which showed a high success rate of $88 \%$ in patients within 42 days [25]. Nonetheless, we have no experience in managing cases whose symptoms have been for over 3 months. Ottesen reported 10 such patients with DAIR with success rate of $60 \%$, which was encouraging [25]. However, it merits further evaluation to see if DAIR could be performed to treat delayed or chronic PJI. 
The third finding of our study was that we showed DAIR had high success rate for Staphylococcal infections. All the 3 cases with staphylococcus aureus infections were successfully treated, and even for the methicillin-resistant staphylococcus, as was seen in our 9 MRSE infections, DAIR completely eradicated infection. Staphylococcal infection has been viewed as a risk factor for failed DAIR, as literature reported high failure rate ranging from $45 \%$ to $76 \%$ (Table 3). One possible explanation is that Staphylococcus is more easier to form biofilm on the surface of implants, as well as its drug-resistance to antibiotics [3]. Thus several researchers have proposed staphylococcal infection to be a surgical contradiction to DAIR, especially for methicillin-resistant staphylococcus [39, 40] [41]. For streptococcal infection, our limited data demonstrated a general satisfactory success rate of $71.4 \%(5 / 7)$, which was still higher than the data from a multi-center study $(35 \%, 11 / 31)[15]$.

Another finding of this study is on the antibiotics regime. In our series, IV antibiotics were generally used for 2 weeks followed by 4 weeks of oral antibiotics, regardless of hip or knee infection. The 2013 guideline of Infectious Diseases Society of America (IDSA) has recommended IV antibiotics for 2-6 weeks followed by oral antibiotics for 3 months for hip infections, and 6 months for knee infections for treating acute PJI [44]. Nonetheless, the optimal duration of antibiotics has been controversial. Various studies have shown a similar infection eradication rate between short-term antibiotics and long-term antibiotics regime [34]. And a recent multi-center randomized trails did show that oral antibiotic therapy was noninferior to intravenous antibiotic therapy when used during the first 6 weeks for complex orthopedic infection [45]. Long-term antibiotics regime brings several adverse effects to the patients, and our study suggest a short-term antibiotics ( 2 weeks IV plus 4 weeks of oral) can be considered for acute PJI. More randomized controlled trials are warranted to address this issue.

There are several limitations of this study that need to be acknowledged. First, same as other reports, this is a retrospective study without comparative controls and with limited sample size. Second, the follow-up period is relatively short, and it's possible that some slow, low-virulence infections might present itself later than our mean 2-year follow-up period. The long-term treatment outcome remains to be evaluated.

\section{Conclusions}

DAIR has high success rate for acute PJI. In selected patients whose symptoms have sustained for over 4 weeks, DAIR could be considered as well. DAIR has high rate of success for staphylococcal infection. mNGS test can be used as a potential tool to identify pathogens in acute PJI.

\section{Abbreviations}

DAIR: debridement, antibiotics and implant retention; PJI: periprosthetic joint infection; ASA: American Society of Anesthesiologists; DM:diabetes mellitus; MSIS: Musculoskeletal Infection Society; CRP: CReactive Protein; ESR: Erythrocyte Sedimentation Rate; WBC: White Blood Cell; mNGS: metagenomic nextgeneration sequencing; ONFH: osteonecrosis of femoral head; AS: ankylosing spondylitis; UKA: unicondylar knee replacement; MRSE: Methicillin-resistant Staphylococcus epidermidis; THA: total hip 
replacement; TKA: total knee replacement; TEA: total elbow replacement; ICM: international concensus meeting; IDSA: Infectious Diseases Society of America

\section{Declarations}

\section{Acknowledgment}

None

\section{Author's contributions:}

C Zhang: literature review, collected data, analyzed data, wrote the manuscript; L He: collected data, analyzed data, wrote the manuscript; X Fang: literature review, collected data, analyzed data; Z Huang: collected data, analyzed data; G Bai: collected data, analyzed data; W Li: collected data, analyzed data; W Zhang: created the concept, designed the study, analyzed data, wrote the manuscript.

\section{Funding}

This work was supported by: Startup Fund for Scientific Research, Fujian Medical University (2018QH1053); Natural Science Foundation of Fujian Province (2018Y4003); Natural Science Foundation of Fujian Province (201810006)

\section{Availability of data and materials}

The datasets used and/or analysed during the current study are available from the corresponding author on reasonable request.

\section{Ethics approval and consent to participate}

This study was approved by the Ethics Committee and Institutional Review Board of our institution (Approval No: [2019] 202). Informed consent was obtained from each patient before the data was collected.

\section{Consent for publication}

Participants gave informed consent for publication. 


\section{Competing Interests}

The authors declare no competing interests.

\section{References}

1. Kurtz S, Lau E, Watson H. Economic burden of periprosthetic joint infection in the United States. J Arthroplasty. 2012;27 8 supplement:61-65 e1.

2. Kurtz SM, Lau EC, Son MS, Chang ET, Zimmerli W, Parvizi J. Are We Winning or Losing the Battle With Periprosthetic Joint Infection: Trends in Periprosthetic Joint Infection and Mortality Risk for the Medicare Population. J Arthroplasty. 2018;33:3238-45.

3. Tsukayama DT, Estrada R, Gustilo RB. Infection after total hip arthroplasty. A study of the treatment of one hundred and six infections. J Bone Joint Surg Am. 1996;78:512-23.

4. Kim Y-H, Choi Y, Kim J-S. Treatment based on the type of infected TKA improves infection control. Clin Orthop Relat Res. 2011;469:977-84.

5. Achermann Y, Stasch P, Preiss S, Lucke K, Vogt M. Characteristics and treatment outcomes of 69 cases with early prosthetic joint infections of the hip and knee. Infection. 2014;42:511-9.

6. Westberg M, Grøgaard B, Snorrason F. Early prosthetic joint infections treated with debridement and implant retention: 38 primary hip arthroplasties prospectively recorded and followed for median 4 years. Acta Orthop. 2012;83:227-32.

7. Van Kleunen JP, Knox D, Garino JP, Lee G-C. Irrigation and debridement and prosthesis retention for treating acute periprosthetic infections. Clin Orthop Relat Res. 2010;468:2024-8.

8. Achermann Y, Vogt M, Spormann C, Kolling C, Remschmidt C, Wüst J, et al. Characteristics and outcome of 27 elbow periprosthetic joint infections: results from a 14-year cohort study of 358 elbow prostheses. Clin Microbiol Infect. 2011;17:432-8.

9. Shanmugasundaram S, Ricciardi BF, Briggs TWR, Sussmann PS, Bostrom MP. Evaluation and Management of Periprosthetic Joint Infection-an International, Multicenter Study. HSS Journal®. 2014;10:36-44.

10. Cobo J, Miguel L, Euba G, Rodríguez D, García-Lechuz JM, Riera M, et al. Early prosthetic joint infection: outcomes with debridement and implant retention followed by antibiotic therapy. Clin Microbiol Infect. 2011;17:1632-7.

11. Zmistowski B, Fedorka CCJ, Sheehan E, Deirmengian G, Austin MS, Parvizi J. Prosthetic joint infection caused by gram-negative organisms. J Arthroplasty. 2011;26:104-8.

12. Azzam KA, Seeley M, Ghanem E, Austin MS, Purtill JJ, Parvizi J. Irrigation and debridement in the management of prosthetic joint infection: traditional indications revisited. J Arthroplasty. 2010;25:1022-7.

13. Zhang $\mathrm{C}$, Yan $\mathrm{CH}$, Chan PK, Ng FY, Chiu KY. Polyethylene Insert Exchange Is Crucial in Debridement for Acute Periprosthetic Infections following Total Knee Arthroplasty. J Knee Surg. 2017;30:36-41. 
14. Fehring TK, Odum SM, Berend KR, Jiranek WA, Parvizi J, Bozic KJ, et al. Failure of irrigation and debridement for early postoperative periprosthetic infection. Clin Orthop Relat Res. 2013;471:250-7.

15. Odum SM, Fehring TK, Lombardi A V, Zmistowski BM, Brown NM, Luna JT, et al. Irrigation and debridement for periprosthetic infections: does the organism matter? J Arthroplasty. 2011;26:114-8.

16. Choi H-R, Von Knoch F, Zurakowski D, Nelson SB, Malchau H. Can implant retention be recommended for treatment of infected TKA? Clin Orthop Relat Res. 2011;469:961-9.

17. Koyonos L, Zmistowski B, Della Valle CJ, Parvizi J. Infection control rate of irrigation and débridement for periprosthetic joint infection. Clin Orthop Relat Res. 2011;469:3043-8.

18. Qasim SN, Swann A, Ashford R. The DAIR (debridement, antibiotics and implant retention) procedure for infected total knee replacement-a literature review. Sicot-J. 2017;3:2.

19. Parvizi J, Zmistowski B, Berbari EF, Bauer TW, Springer BD, Della Valle CJ, et al. New Definition for Periprosthetic Joint Infection. From the Workgroup of the Musculoskeletal Infection Society. Clin Orthop Relat Res. 2011;469:2992-4.

20. Zimmerli W, Trampuz A, Ochsner PE. Prosthetic-Joint Infections. N Engl J Med. 2004;351:1645-54.

21. CROCKARELL JR, Hanssen AD, Osmon DR, Morrey BF. Treatment of Infection with Débridement and Retention of the Components following Hip Arthroplasty. J Bone Jt Surg. 1998;80:1306-13.

22. Theis JC. Implant retention in infected joint replacements: a surgeon's perspective. Int J Artif Organs. 2008;31:804-9.

23. Marculescu CE, Berbari EF, Hanssen AD, Steckelberg JM, Harmsen SW, Mandrekar JN, et al. Outcome of prosthetic joint infections treated with debridement and retention of components. Clin Infect Dis. 2006;42:471-8.

24. Aboltins C, Dowsey MM, Peel T, Lim WK, Parikh S, Stanley P, et al. Early prosthetic hip joint infection treated with debridement, prosthesis retention and biofilm-active antibiotics: functional outcomes, quality of life and complications. Intern Med J. 2013;43:810-5.

25. Ottesen CS, Troelsen A, Sandholdt H, Jacobsen S, Husted H, Gromov K. Acceptable Success Rate in Patients With Periprosthetic Knee Joint Infection Treated With Debridement, Antibiotics, and Implant Retention. J Arthroplasty. 2019;34:365-8.

26. Byren I, Bejon P, Atkins BL, Angus B, Masters S, McLardy-Smith P, et al. One hundred and twelve infected arthroplasties treated with "DAIR"(debridement, antibiotics and implant retention): antibiotic duration and outcome. J Antimicrob Chemother. 2009;63:1264-71.

27. Klouche S, Lhotellier L, Mamoudy P. Infected total hip arthroplasty treated by an irrigationdebridement/component retention protocol. A prospective study in a 12-case series with minimum 2 years' follow-up. Orthop Traumatol Surg Res. 2011;97:134-8.

28. Waagsbø B, Sundøy A, Martinsen TML, Nymo LS. Treatment results with debridement and retention of infected hip prostheses. Scand J Infect Dis. 2009;41:563-8.

29. Segawa H, Tsukayama DDT, Kyle RRF, Becker DA, Gustilo RB. Infection After Total Knee Arthroplasty. A Retrospective Study of the Treatment of Eighty-One Infections. J Bone Jt Surg. 1999;81:1434-45. 
30. Silva M, Tharani R, Schmalzried TP. Results of direct exchange or debridement of the infected total knee arthroplasty. Clin Orthop Relat Res. 2002;404:125-31.

31. Romanò CL, Manzi G, Logoluso N, Romanò D. Value of debridement and irrigation for the treatment of peri-prosthetic infections. A systematic review. Hip Int. 2011;22:S19-24.

32. Konigsberg BS, Della Valle CJ, Ting NT, Qiu F, Sporer SM. Acute hematogenous infection following total hip and knee arthroplasty. J Arthroplasty. 2014;29:469-72.

33. Sherrell JC, Fehring TK, Odum S, Hansen E, Zmistowski B, Dennos A, et al. The Chitranjan Ranawat Award: fate of two-stage reimplantation after failed irrigation and debridement for periprosthetic knee infection. Clin Orthop Relat Res. 2011;469:18-25.

34. Chotanaphuti T, Courtney PM, Fram B, In den Kleef NJ, Kim T-K, Kuo F-C, et al. Hip and Knee Section, Treatment, Algorithm: Proceedings of International Consensus on Orthopedic Infections. J Arthroplasty. 2019;34:S393-7.

35. Reis-Filho JS. Next-generation sequencing. Breast Cancer Res. 2009;11 Suppl 3:S12.

36. Street TL, Sanderson ND, Atkins BL, Brent AJ, Cole K, Foster D, et al. Molecular Diagnosis of Orthopedic-Device-Related Infection Directly from Sonication Fluid by Metagenomic Sequencing. J Clin Microbiol. 2017;55:2334-47.

37. Huang Z, Zhang C, Li W, Fang X, Wang Q, Xing L, et al. Metagenomic next-generation sequencing contribution in identifying prosthetic joint infection due to Parvimonas micra: a case report. J Bone Jt Infect. 2019;4:50-5.

38. Huang Z, Zhang C, Fang X, Li W, Zhang C, Zhang W, et al. Identification of musculoskeletal infection with non-tuberculous mycobacterium using metagenomic sequencing. J Infect. 2019;78:158-69.

39. Bradbury T, Fehring TK, Taunton M, Hanssen A, Azzam K, Parvizi J, et al. The fate of acute methicillin-resistant Staphylococcus aureus periprosthetic knee infections treated by open debridement and retention of components. J Arthroplasty. 2009;24:101-4.

40. Walls RJ, Roche SJ, O'Rourke A, McCabe JP. Surgical site infection with methicillin-resistant Staphylococcus aureus after primary total hip replacement. J Bone Joint Surg Br. 2008;90:292-8.

41. Deirmengian C, Greenbaum J, Lotke PA, Booth RE, Lonner JH. Limited success with open debridement and retention of components in the treatment of acute Staphylococcus aureus infections after total knee arthroplasty. J Arthroplasty. 2003;18:22-6.

42. Lora-Tamayo J, Murillo O, Iribarren JA, Soriano A, Sánchez-Somolinos M, Baraia-Etxaburu JM, et al. A Large Multicenter Study of Methicillin-Susceptible and Methicillin-Resistant Staphylococcus aureus Prosthetic Joint Infections Managed With Implant Retention. Clin Infect Dis. 2013;56:182-94.

43. Gardner J, Gioe TJ, Tatman P. Can this prosthesis be saved?: implant salvage attempts in infected primary TKA. Clin Orthop Relat Res. 2011;469:970-6.

44. Osmon DR, Berbari EF, Berendt AR, Lew D, Zimmerli W, Steckelberg JM, et al. IDSA. Clin Infect Dis. 2013;56:e1-25. 
45. Li H-K, Rombach I, Zambellas R, Walker AS, McNally MA, Atkins BL, et al. Oral versus Intravenous Antibiotics for Bone and Joint Infection. N Engl J Med. 2019;380:425-36.

\section{Tables}

Due to technical limitations, tables are only available as a download in the supplemental files section

\section{Figures}

\section{Figure 1. Bacteriology of included cases with positive culture/mNGS.}

\section{Total $=22$}

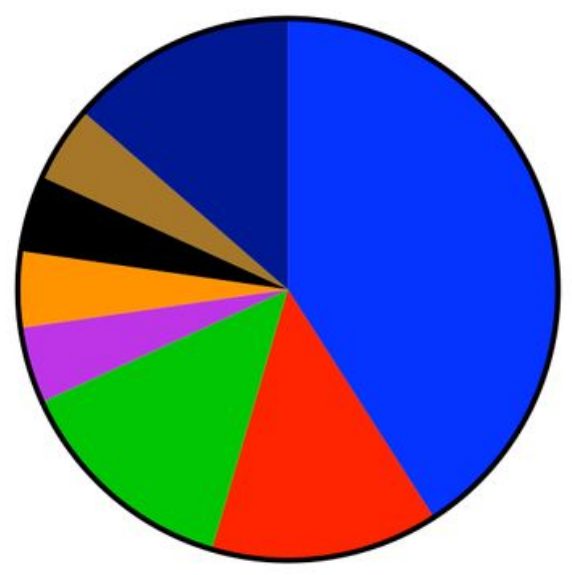

$\square$ MRSE

$\square$ Staphylococcus aureus

$\square$ Streptococcus agalactiae

$\square$ Staphylococcus haemolyticus

$\square$ Streptococcus agalactiae subsp. equisimilis

- Streptococcus gordonii

$\square$ Bacteroides

$\square$ mixed infections

Figure 1

Bacteriology of included cases with positive culture/mNGS results.

\section{Supplementary Files}

This is a list of supplementary files associated with this preprint. Click to download.

- supplement1.docx 
- supplement2.docx

- supplement3.docx 